\title{
ON THE SOLUTION OF A CERTAIN CLASS OF DIFFERENCE OR DIFFERENTIAL EQUATIONS.
}

[American Journal of Mathematies, IV. (1881), pp. 260-265.]

Casting my eye over Mr Moulton's valuable edition of Boole's Treatise on Finite Differences (see pp. 229-231), I was gratified to find that he had embalmed in it a solution that I had given* many years ago, of an equation in differences, of the simple but very general form expressed by equating to zero or to $P m^{x}$ the persymmetrical determinant

$$
\left|\begin{array}{cccc}
u_{x} & u_{x+1} & \ldots & u_{x+i} \\
u_{x+1} & u_{x+2} & \ldots & u_{x+i+1} \\
u_{x+2} & u_{x+3} & \ldots & u_{x+i+2} \\
\cdots \cdots \cdots \cdots \cdots \cdots & \cdots \cdots \cdots \cdots \\
\cdots \cdots \cdots \cdots \cdots \cdots \cdots & \cdots \cdots \cdots \cdots \\
u_{x+i} & u_{x+i+1} & \ldots & u_{x+2 i}
\end{array}\right|
$$

which is of the $i$ th degree and $2 i$ th order.

To fix the ideas, let us consider the simple case

$$
u_{x} u_{x+2}-u_{x+1}^{2}=P m^{x},
$$

of which, when $P=0$, the solution is $u_{x}=A \alpha^{x}, A$ and $\alpha$ being both arbitrary, but for $P$ not zero is expressed by $u_{x}= \pm\left(A \alpha^{x}+B \beta^{x}\right)$ with the conditions

$$
\alpha \beta=m, \quad A B(\alpha-\beta)^{2}=P
$$

which solution as an obiter dictum I may remark may easily be converted into the simpler and more explicit form

$$
(\sin \beta)^{2} u_{x}^{2}+P\{\sin (\alpha+\beta x)\}^{2} m^{x-1}=0
$$

where $\alpha, \beta$ are arbitrary constants.

If we proceed now to verify the solution in its original form, we shall immediately be led to perceive a certain generalization which the given equation may be made to undergo without ceasing to be soluble-the solution however becoming narrowed from a general to a special one: whether particular or singular I shall not discuss.

[* This Reprint, Vol. II., pp. 308, 313.] 
If we write $u_{x}=A \alpha^{x}+B \beta^{x}$, the determinant becomes

$$
\begin{array}{ll}
A \alpha^{x}+B \beta^{x}, & A \alpha^{x+1}+B \beta^{x+1} \\
A \alpha^{x+1}+B \beta^{x+1}, & A \alpha^{x+2}+B \beta^{x+2}
\end{array}
$$

which is equal to $A B(\alpha-\beta)^{2}(\alpha \beta)^{x}$; this is the verification spoken of: but, as a consequence, it is apparent that we must have

$$
\begin{aligned}
& \left|\begin{array}{l}
A \alpha^{x}+B \beta^{x}+C \gamma^{x}, \quad A \alpha^{x+1}+B \beta^{x+1}+C \gamma^{x+1} \\
A \alpha^{x+1}+B \beta^{x+1}+C \gamma^{x+1}, \quad A \alpha^{x+2}+B \beta^{x+2}+C \gamma^{x+2}
\end{array}\right| \\
& =A B(\alpha-\beta)^{2}(\alpha \beta)^{x}+B C(\beta-\gamma)^{2}(\beta \gamma)^{x}+C A(\gamma-\alpha)^{2}(\gamma \alpha)^{x} .
\end{aligned}
$$

Hence we can solve the equation

$$
u_{x} u_{x+2}-u_{x+1}^{2}=P l^{x}+Q m^{x}+R n^{x},
$$

namely, we may write $u_{x}+A \alpha^{x}+B \beta^{x}+C \gamma^{x}=0$,

where

$$
\beta \gamma=l, \quad \gamma \alpha=m, \quad \alpha \beta=n,
$$

$$
A B(\alpha-\beta)^{2}=R, \quad B C(\beta-\gamma)^{2}=P, \quad C A(\gamma-\alpha)^{2}=Q,
$$

that is to say $\alpha=\sqrt{ }\left(\frac{m n}{l}\right), \quad \beta=\sqrt{ }\left(\frac{n l}{m}\right), \quad \gamma=\sqrt{ }\left(\frac{l m}{n}\right)$,

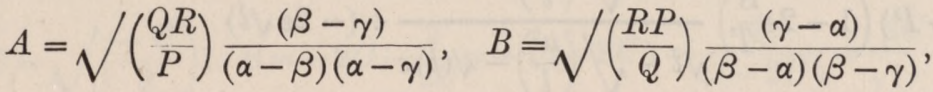

$$
\begin{aligned}
& C=\sqrt{ }\left(\frac{P Q}{R}\right) \frac{(\alpha-\beta)}{(\gamma-\alpha)(\gamma-\beta)} ;
\end{aligned}
$$

or calling

$$
\begin{gathered}
\sqrt{ }(l m n)=g, \quad \sqrt{ }(P Q R)=G, \\
\alpha=\frac{g}{l}, \quad \beta=\frac{g}{m}, \quad \gamma=\frac{g}{n}, \\
A=\frac{G}{g} \cdot \frac{(n-m) l^{2}}{(l-m)(l-n) P}, \quad B=\frac{G}{g} \cdot \frac{(l-n) m^{2}}{(m-n)(m-l) Q}, \\
C=\frac{G}{g} \cdot \frac{(m-l) n^{2}}{(n-l)(n-m) R} .
\end{gathered}
$$

The result therefore in its rational unambiguous form is

$$
P Q R(l m-m n)^{2}(m n-n l)^{2}(n l-l m)^{2}(l m n)^{2 x-1} u_{x}^{2}=\left\{\Sigma(l m-l n) Q R(m n)^{x}\right\}^{2} .
$$

When any of the quantities $P, Q, R$ vanish, or any of the quantities $l, m, n$ vanish or become equal to one another, the solution fails.

We shall, however, easily obtain a compensatory form of equation supplying the place of two of the exponentials, and another supplying the place of all three becoming identical, and the solution of these substituted forms may be deduced from that of the original form of the equation. 
Thus, first, let

$$
\left.\begin{array}{ll}
m=(1+\epsilon) \mu, & n=(1-\epsilon) \mu \\
Q=\frac{1}{2}\left(S+\frac{T}{\epsilon}\right), & R=\frac{1}{2}\left(S-\frac{T}{\epsilon}\right)
\end{array}\right\} \text { where } \epsilon \text { is an infinitesimal. }
$$

Then the equation becomes

$$
u_{x} u_{x+2}-u_{x+1}^{2}=P l^{x}+S \mu^{x}+T x \mu^{x}
$$

and the solution in its unreduced form is

$$
u_{x}=A \alpha^{x}+B \beta^{x}+C \gamma^{x},
$$

where

$$
\alpha=\sqrt{\frac{\mu^{2}}{l},} \quad \beta=(1-\epsilon) \sqrt{ } l, \quad \gamma=(1+\epsilon) \sqrt{ } l,
$$

and

$$
\begin{aligned}
& A=\sqrt{ }\left(\frac{P R}{Q}\right) \frac{\beta-\gamma}{(\alpha-\beta)(\alpha-\gamma)}=\frac{T}{\sqrt{ }(-P)} \frac{\sqrt{ } l}{\left\{\sqrt{ }\left(\frac{\mu^{2}}{l}\right)-\sqrt{ } l\right\}^{2}}=\frac{T}{\sqrt{ }(-P)} \cdot \frac{l^{\frac{3}{2}}}{(\mu-l)^{2}} \\
& B=\sqrt{ }\left(\frac{P R}{Q}\right) \frac{\gamma-\alpha}{(\beta-\alpha)(\beta-\gamma)} \\
& =\sqrt{ }(-P)\left(1-2 \epsilon \frac{S}{T}\right) \frac{\sqrt{ } l-\sqrt{ }\left(\frac{\mu^{2}}{l}\right)+\sqrt{ }(l) \epsilon}{\sqrt{ } l-\sqrt{ }\left(\frac{\mu^{2}}{l}\right)-\sqrt{ }(l) \epsilon} \div(-2 \epsilon \sqrt{ } l) \\
& =\sqrt{ }(-P)\left\{\frac{-1}{2 \sqrt{ }(l) \epsilon}+\frac{S}{T \sqrt{ } l}-\frac{\sqrt{ } l}{l-\mu}\right\} \\
& C=\sqrt{ }(-P)\left\{\frac{1}{2 \sqrt{ }(l) \epsilon}+\frac{S}{T \sqrt{ } l}-\frac{\sqrt{ } l}{l-\mu}\right\} \text {. }
\end{aligned}
$$

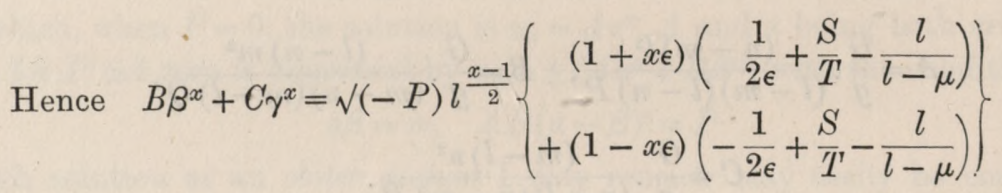

$$
\begin{aligned}
& =\sqrt{ }(-P)\left\{2\left(\frac{S}{T}-\frac{l}{l-\mu}\right)+x\right\} l^{\frac{x-1}{2}} \\
& \text { so that } \quad \sqrt{ }(-l P) u_{x}=T\left(\frac{l}{\mu-l}\right)^{2}\left(\frac{\mu^{2}}{l}\right)^{\frac{x}{2}}-P\left(\frac{2 S}{T}-\frac{2 l}{l-\mu}+x\right) l^{\frac{x}{2}} \\
& \text { or } \\
& P l^{x+1} u_{x}^{2}+\left\{T\left(\frac{l}{\mu-l}\right)^{2} \mu^{x}-P\left(\frac{2 S}{T}+\frac{2 l}{\mu-l}+x\right) l^{x}\right\}^{2}=0
\end{aligned}
$$

will satisfy the given equation

$$
u_{x} u_{x+2}-u_{x+1}^{2}=P l^{x}+S \mu^{x}+T x \mu^{x} .
$$

When $T=0$ the solution fails, as we know à priori it ought to do. 
When $S=0$ it takes the form

$$
P l^{x+1} u_{x}{ }^{2}+\left\{T\left(\frac{l}{\mu-l}\right)^{2} \mu^{x}-P\left(\frac{2 l}{\mu-l}+x\right) l^{x}\right\}^{2}=0 .
$$

We might, by an analogous process, writing $(1+\epsilon),(1+\rho \epsilon),\left(1+\rho^{2} \epsilon\right)$ in lieu of $l, m, n$, and giving $P, Q, R$ appropriate values involving $\epsilon^{2}$ as well as $\epsilon$, render $\Sigma P l^{x}$ a finite function of the form $\left(S+T x+U x^{2}\right) \lambda^{x}$, and deduce the solution of $u_{x} u_{x+2}-u_{x+1}^{2}=\left(S+T x+U x^{2}\right) \lambda^{x}$ as a particular case of the solution of the general equation. But as we can easily see that the unreduced form of the solution must be $u_{x}=\lambda^{\frac{x}{2}}\left(A+B x+C x^{2}\right)$, it will be easier to find $A, B, C$ immediately from the equation

$$
\left|\begin{array}{ll}
A+B x+C x^{2} & A+B(x+1)+C(x+1)^{2} \\
A+B(x+1)+C(x+1)^{2} & A+B(x+2)+C(x+2)^{2}
\end{array}\right|
$$

or

or

Hence $\quad-2 C^{2}=U, \quad-2 B C-4 C^{2}=T, \quad 2 A C-(B+C)^{2}=S$.

Hence

$$
\begin{aligned}
& C= \sqrt{\left(\frac{-U}{2}\right), B=-2 C-\frac{T}{2 C}=-\sqrt{ }(-2 U)-\frac{T}{\sqrt{ }(-2 U)}=\frac{2 U+T}{\sqrt{ }(-2 U)}}, \\
& A=\frac{S+(B+C)^{2}}{2 C}=\frac{S+\left\{\sqrt{\left.\left(\frac{-U}{2}\right)+\frac{T}{\sqrt{ }(-2 U)}\right\}^{2}}\right.}{\sqrt{ }(-2 U)}=\frac{-2 S U+(T+U)^{2}}{-2 U \sqrt{ }(-2 U)} \\
& \text { or } \quad 8 U^{3} u_{x}^{2}+\left\{2 U^{2} x^{2}+\left(4 U^{2}-2 U T\right) x+2 S U-(T+U)^{2}\right\} \lambda^{x}=0
\end{aligned}
$$

is the required primitive of the given equation.

The method may obviously be extended to any equation of the given form: that is to say when the persymmetrical determinant which it contains is of the degree $i$ and is equated to $(i+1)$ multiples of exponentials each of the form $P l^{x}$ an integral of it can be found, and if these $i$ exponentials be subdivided into partial groups of $\epsilon, \epsilon^{\prime}, \epsilon^{\prime \prime} \ldots$ terms in a group, then instead of the $\epsilon$ multiples of exponentials belonging to any group may be substituted

$$
\left(P_{1}+P_{2} x+P_{3} x^{2}+\ldots+P_{\mathrm{e}} x^{\epsilon-1}\right) l^{x}
$$

and the solution of the equation so modified may be deduced from the solution first mentioned as a particular case thereof.

It will be sufficient for all reasonable purposes of illustration briefly to consider the case of

$$
\left|\begin{array}{lll}
u_{x} & u_{x+1} & u_{x+2} \\
u_{x+1} & u_{x+2} & u_{x+3} \\
u_{x+2} & u_{x+3} & u_{x+4}
\end{array}\right|=P l^{x}+Q m^{x}+R n^{x}+S p^{x}
$$


An integral of this may be found by writing

where

$$
u_{x}=A \alpha^{x}+B \beta^{x}+C \gamma^{x}+D \delta^{x},
$$

$$
\begin{aligned}
& \beta \gamma \delta=l, \quad \alpha \gamma \delta=m, \quad \alpha \beta \delta=n, \quad \alpha \beta \gamma=p, \\
& B C D \zeta(\beta, \gamma, \delta)=P, \quad A C D \zeta(\alpha, \gamma, \delta)=Q, \quad A B D \zeta(\alpha, \beta, \delta)=R, \\
& A B C \zeta(\alpha, \beta, \gamma)=S \text {, }
\end{aligned}
$$

$\zeta$ meaning the product of the squared differences of the letters which it governs. We have thus

$$
\alpha=\frac{g}{l}, \quad \beta=\frac{g}{m}, \quad \gamma=\frac{g}{n}, \quad \delta=\frac{g}{p},
$$

where

$$
g=\sqrt[3]{ }(\operatorname{lm} n p)
$$

and

$$
A^{3} B^{3} C^{3} D^{3}[\zeta(\alpha, \beta, \gamma, \delta)]^{2}=P Q R S,
$$

so that writing

$$
G=\left\{\frac{P Q R S}{[\zeta(\alpha, \beta, \gamma, \delta)]^{2}}\right\}^{\frac{1}{3}}=\frac{1}{g^{8}} \frac{(P Q R S)^{\frac{1}{3}}}{\left\{\zeta\left(\frac{1}{l}, \frac{1}{m}, \frac{1}{n}, \frac{1}{p}\right)\right\}^{\frac{2}{3}}},
$$

$$
A=\zeta(\beta, \gamma, \delta) \frac{G}{P} ; \quad B=\zeta(\alpha, \gamma, \delta) \frac{G}{Q} ; \quad C=\zeta(\alpha, \beta, \delta) \frac{G}{R} ; \quad D=\zeta(\alpha, \beta, \gamma) \frac{G}{S}
$$

and thus

$$
(P Q R S)^{2}(l m n p)^{8}\left\{\zeta\left(\frac{1}{l}, \frac{1}{m}, \frac{1}{n}, \frac{1}{p}\right)\right\}^{2} u_{x}^{3}=(l m n p)^{x}\left\{\Sigma Q R S \zeta(\beta, \gamma, \delta) l^{-x}\right\}^{3}
$$

or

$$
\left\{P Q R S \zeta\left(\frac{1}{l}, \frac{1}{m}, \frac{1}{n}, \frac{1}{p}\right)\right\}^{2} u_{x}^{3}=(\operatorname{lm} n p)^{x-2}\left\{\Sigma Q R S \zeta\left(\frac{1}{m}, \frac{1}{n}, \frac{1}{p}\right) l^{-x}\right\}^{3} .
$$

It is scarcely necessary to add that all the above conclusions continue to hold, when, on the left hand side of the equation for $u_{x+h}$ we write $\left(\frac{d}{d x}\right)^{h} y$ and at the same time for any exponential $l^{x}$ on the right hand side substitute $e^{l x}$.

Thus for instance we may in general find an integral of

or again of

$$
y y^{\prime \prime}-y^{\prime 2}=A e^{h x}+B e^{k x} \cos (\alpha x+\beta)
$$

$$
\left(y y^{\prime \prime}-y^{\prime 2}\right) y^{\prime \prime \prime \prime}-y\left(y^{\prime \prime \prime}\right)^{2}+2 y^{\prime} y^{\prime \prime} y^{\prime \prime \prime}-y^{\prime \prime 3}=A e^{h x} \cos (\alpha x+\beta)+B e^{k x} \cos (\gamma x+\delta) \text {. }
$$

